\title{
Study of comparative surgical exposure to the petroclival region using patient-specific, petroclival meningioma virtual reality models
}

\author{
${ }^{*}$ Walter C. Jean, MD, ${ }^{1}$ Yang Yang, MD, ${ }^{2}$ Aneil Srivastava, BS, ${ }^{3}$ Alexander X. Tai, MD, ${ }^{4}$ \\ Aalap Herur-Raman, MS, BME, ${ }^{5}$ H. Jeffrey Kim, MD, ${ }^{6}$ Da Li, MD, ${ }^{2}$ and Zhen Wu, MD, PhD ${ }^{2}$ \\ 1Department of Neurosurgery and ${ }^{5} \mathrm{~S} c h o o l$ of Medicine and Health Sciences, George Washington University; Departments \\ of ${ }^{4}$ Neurosurgery and ${ }^{6}$ Otolaryngology, Georgetown University, Washington, DC; ${ }^{2}$ Beijing Tiantan Hospital, Capital Medical \\ University, Beijing, People's Republic of China; and ${ }^{3}$ Surgical Theater, Mayfield Village, Ohio
}

\begin{abstract}
OBJECTIVE Despite advancement of surgical techniques, the attachments of petroclival meningiomas near the central clival depression (CCD) remain difficult to visualize. With existing methods, the amount of tumor near the CCD that is inaccessible through various approaches cannot be compared. Tumors distort the brainstem, changing the size of the operative corridor for some but not all approaches; therefore, using cadavers with normal posterior fossae makes it impossible to compare different approaches to the tumor. The authors used virtual reality (VR) models created from the imaging data of patients to compare various surgical approaches that have otherwise been incomparable in previous studies.
\end{abstract}

METHODS CT and MRI data obtained in 15 patients with petroclival meningiomas were used to create anatomically accurate 3D VR models. For each model, various surgical approaches were performed, and the surgical freedom to 6 targets of the regions were measured. Furthermore, portions of the tumor that were visually blocked by the brainstem or bony structures were segmented and recorded as blinded volumes for comparison.

RESULTS The extended retrosigmoid approach generated excellent exposure of the petroclival region, but for most specimens, there was inaccessible tumor volume adjacent to the brainstem (mean $641.3 \mathrm{~mm}^{3}, \mathrm{SE} 161.8$ ). In contrast, the brainstem sides of the tumors were well-visualized by all the transpetrosal approaches. The blinded volume of the tumor was largest for the retrolabyrinthine approach, and this was statistically significant compared with all other approaches (mean $2381.3 \mathrm{~mm}^{3}$, SE 185.4).

CONCLUSIONS The authors performed a novel laboratory study by using patient CT and MRI data to generate 3D virtual models to compare surgical approaches. Since it is impossible to perform various approaches in separate surgeries in patients for comparison, VR represents a viable alternative for such comparative investigations.

https://thejns.org/doi/abs/10.3171/2021.5.FOCUS201036

KEYWORDS meningioma; petroclival; virtual reality; surgical exposure

A CCESSING the petroclival region remains a challenge to skull base surgeons. Almefty et al. defined "true" petroclival meningiomas as those "originating at the upper two-thirds of the clivus medial to the fifth cranial nerve." To improve exposure to these structures, transpetrosal approaches were designed to provide the surgeon a more anterolateral line of sight to better visualize these entangled nerves, arteries, and brainstem..$^{2-5}$

In their landmark study, Abdel Aziz et al. divided the petroclival region into three zones and designated the best approach to each zone. ${ }^{6}$ The authors discovered that the central clival depression (CCD) is not accessible without drilling through the cochlea, and the greater the depth of the CCD, the harder it is to reach. ${ }^{6}$ This conclusion was drawn from cadaveric dissections, but in patients with large tumors that expand the corridor, exposure may be altered. Unfortunately, it is impossible to use patients to compare exposure from various approaches, as neither

ABBREVIATIONS AP = anterior petrosectomy; $C C D=$ central clival depression; $I A C=$ internal acoustic canal; $P C A=$ petroclival angle; $P C F=$ petroclival fissure; $R L=$ retrolabyrinthine; $\mathrm{RS}$ = retrosigmoid; SF = surgical freedom; SRP = Surgical Planner; $T L=$ translabyrinthine; $V R=$ virtual reality.

SUBMITTED December 3, 2020. ACCEPTED May 14, 2021.

INCLUDE WHEN CITING DOI: 10.3171/2021.5.FOCUS201036.

* W.C.J. and Y.Y. contributed equally to this study and share first authorship. 
bone nor tumor have "reset" buttons, and patients can only have one surgery.

We used the CT and MRI data obtained in 15 patients with large, "true" petroclival meningiomas to create anatomically accurate $3 \mathrm{D}$ virtual reality (VR) models. These resettable models allowed us to perform various surgical approaches in VR and to compare measurements of the surgical freedom (SF) to regional targets, and the amount of tumor that was obscured by bone or brainstem.

\section{Methods \\ VR Technology}

In this study, the Surgical Theater Precision VR (Surgical Theater, Inc.) system, Surgical Planner (SRP) 7.4.0, was used. DICOM files of CT and MR images were loaded and reconstructed into a volumetric data set. The coregistration of the scans was rigid, without scaling or distortions, and dimensional data were preserved. A patented algorithm combined the volume rendering with polygon rendering to generate the $3 \mathrm{D}$ model. As previously reported, ${ }^{7-9}$ this VR system has undergone rigorous testing for $\mathrm{K} 1$ clearance of new devices by US FDA requirements. This testing ensured that the size of an object measured in this VR system was within $1 \mathrm{~mm}$ of the physical size of the same object using calipers on a physical phantom. The accuracy of the models in morphometric studies of skull base approaches has also been verified. ${ }^{7-9}$

The databanks at Beijing Tiantan Hospital and George Washington University were queried for patients with large petroclival meningiomas that fit the inclusion criteria. Patients were included if they 1) had a "true" petroclival meningioma that completely traversed the upper two-thirds of the clivus, ${ }^{1,6}$ spanning the top of zone 1 to the bottom of zone 2 (in addition to other areas); and 2) had preoperative thin-cut CTA $(<1 \mathrm{~mm})$ and MRI studies. Fifteen patients were identified, all from Tiantan Hospital. The imaging study sets were anonymized, entered into the study without any associated clinical or demographic information, and used to generate the VR models.

\section{Labeling and Identifying Structures and Targets}

In DICOM, the anatomical structures ipsilateral to the tumor were segmented so that they were visible in VR. These structures were 1) the transverse/sigmoid sinus, 2) cochlea, 3) labyrinth, and 4) mastoid segment of the facial nerve. The tumor was likewise segmented so that it could be separated into zones based on the clivus: ${ }^{6}$ zone 1 , infratentorial, from the dorsum sella to the center of the internal acoustic canal (IAC); zone 2, the center of the IAC to the center of the jugular foramen; and zone 3 , below the jugular foramen. The tumor above zone 1 and in the middle fossa was also segmented.

For SF measurements, markers were placed at 6 targets: the middle of zones 1 and 2 at the ipsilateral petroclival fissure (PCF), midline clivus, and basilar artery (Fig. 1A and B). Markers were also placed at the opening of the IAC and foramen ovale as visual landmarks to guide drilling. Finally, since the brainstem can limit SF and impede visibility to some areas, we segmented the brainstem and considered it an immovable structure.

\section{The Craniotomies}

Virtual craniotomies were performed using the Surgical Theater "drill." With a VR headset and hand controllers, it allows the surgeon to remove voxels from the underlying scans, simulating bone removal.

\section{Retrosigmoid}

Only the "extended" retrosigmoid (RS) craniotomy was used. ${ }^{10-12} \mathrm{~A}$ burr hole was made at the junction of the transverse/sigmoid sinus. The craniotomy extended inferiorly from the transverse/sigmoid sinus junction and stopped $15 \mathrm{~mm}$ above the condylar fossa and posteriorly $20 \mathrm{~mm}$ from the sigmoid sinus. At the anterolateral edge, the bone opening "hugged" the sigmoid sinus, exposing 2 $\mathrm{mm}$ of its posterior edge.

\section{Retrolabyrinthine Posterior Petrosectomy}

Drilling of the petrosectomies was done by a single surgeon (W.C.J.), in collaboration with an experienced neurotologist (H.J.K.). This started with a mastoidectomy, and anteriorly, bone was removed up the posterior lip of the external acoustic meatus. After the mastoid segment of the facial nerve was skeletonized, the bone around the bony labyrinth and over the posterior fossa was eliminated. Anterior to the superior and posterior semicircular canals, bone was removed, stopping short of the IAC. Superiorly, the bone over the tegmen was removed.

\section{Translabyrinthine Posterior Petrosectomy}

Proceeding beyond where the above approach stopped, a total labyrinthectomy was performed. The posterior portion of the IAC was unroofed, leaving approximately onethird of the canal anterior to the facial and vestibulocochlear nerve untouched. Inferiorly, more of the retrofacial bone above the jugular bulb was removed.

\section{Anterior Petrosectomy}

This was performed as an addition to the retrolabyrinthine (RL) opening, as is commonly done and called the combined transpetrosal approach.,13 Starting from the superoanterior tip of the mastoidectomy, a rectangular middle fossa craniotomy was made extending $15 \mathrm{~mm}$ anterior to the root of the zygoma and $20 \mathrm{~mm}$ superiorly. For the drilling of the Kawase quadrilateral, the line between the foramen ovale and the root entry zone of the trigeminal nerve served as the anterior margin. Since the greater superficial petrosal nerve is not visible on DICOM or the VR models, the line connecting the foramen ovale and the IAC in the sagittal plane was used as the lateral margin.

\section{Measurements: Angles and Areas}

Measurements of the petroclival angle (PCA) ${ }^{6,14}$ and SF of each approach were made according to previously described methods. ${ }^{15}$ Four $10-\mathrm{cm}$ posts, simulating the shaft of microsurgical instruments, were placed at each target for each approach (Fig. 1C). Each target had a superior, posterior, anterior, and inferior post, and the posts were extended outward from the target until restricted by the bony edge or the brainstem. The area of the quadrilateral generated by connecting the distal ends of each 

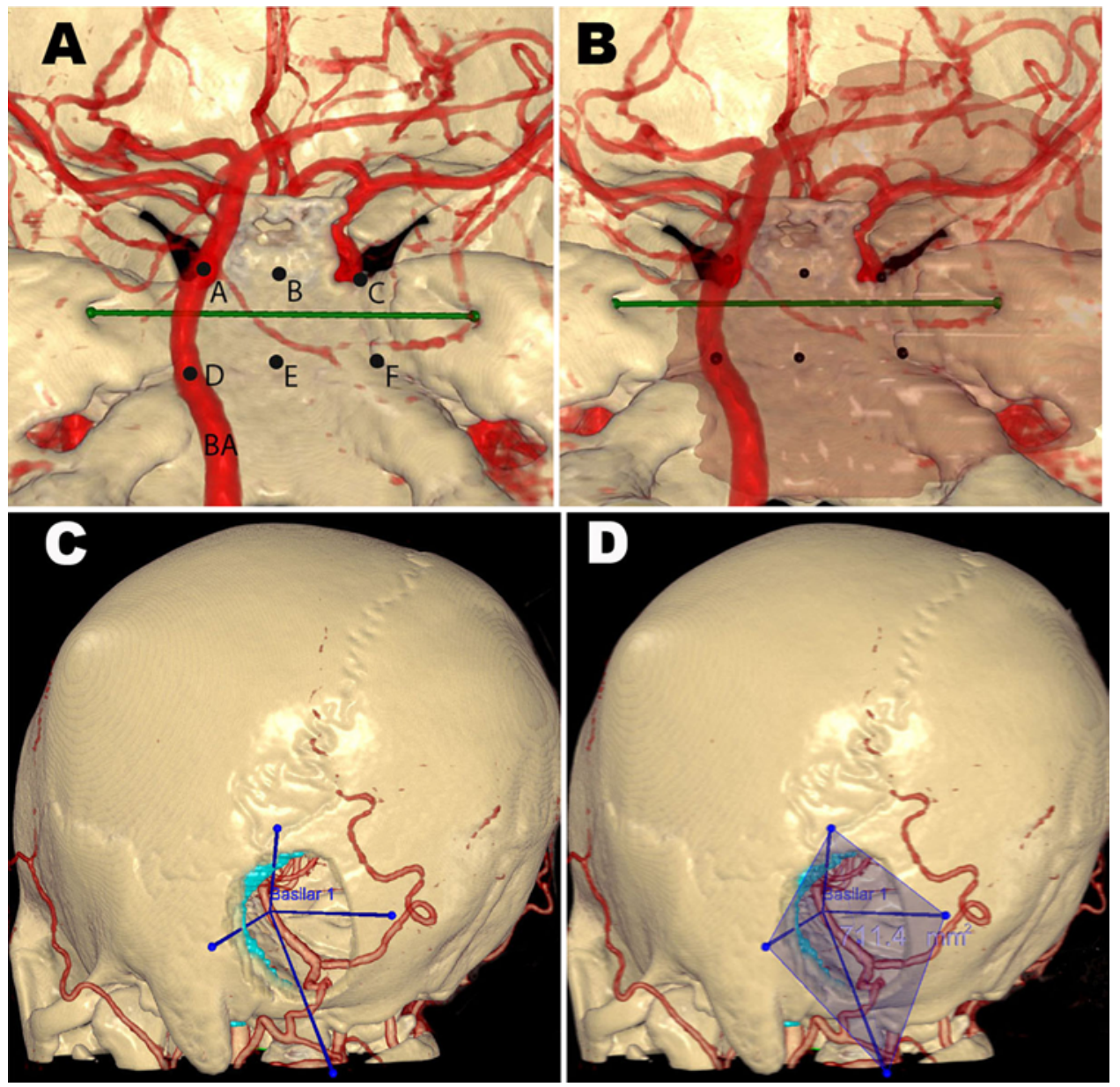

FIG. 1. A: For SF measurements, markers were placed at 6 targets. In this posterior-to-anterior view of the clivus, the targets are: middle of zone 1 at the basilar artery (BA; $A)$, midline clivus $(B)$, ipsilateral PCF $(C)$, as well as the middle of zone 2 at the basilar artery $(D)$, midline clivus $(E)$, and ipsilateral PCF $(F)$. The green line from one IAC to the other demarcates the separation of zones 1 and 2. B: For the same specimen, the tumor is shown in semitransparent fashion to elucidate its relationship with the targets. C: Left-sided extended RS approach with exposure of the inferior portion of the transverse sinus and posterior portion of the sigmoid sinus (light blue). Four 10-cm posts are depicted extending from the basilar artery target in zone 1. D: Quadrilateral using the vertexes of the posts measures the SF of RS to the basilar artery target.

post was measured in VR as the SF (Fig. 1D). Anterior petrosectomy (AP) was considered an add-on for RL in the combined transpetrosal approach, but the SFs for AP were measured only through the subtemporal corridor as stand-alone measurements.

\section{Blinded Volumes}

Similar posts of variable lengths were also placed along the line of sight at the bony margins of each opening (Fig. $2 \mathrm{~A}$ and $\mathrm{B})$. The brainstem is considered untouchable and immovable, and lines of sight were placed along its margins where relevant (Fig. 2D). Since the sigmoid sinus is considered compressible, or at least retractable, by most surgeons, the relevant lines of sight were place up to $1 \mathrm{~mm}$ into the sigmoid sinus (Fig. 2B). The tumor volume behind these lines of sight in zones 1 and 2 were segmented (Fig. $2 \mathrm{C}$ and $\mathrm{E})$. Before recording these volumes as "blinded," the VR models were rotated through all relevant angles in all three dimensions in a process that simulates the an- gulations of the microscope during surgery. Any portions of the segmented volume that were visualized during this rotational examination were erased, and the remainder was recorded as the blinded volume. These methods are summarized in Video 1.

VIDEO 1. Summary of the experimental method for measurement of blinded volumes. The lines of sight were removed for clarity. Dark red indicates zone 1; bright red indicates zone 2; bright green is the brainstem; dark green is the semicircular canals; yellow is the facial nerve (mastoid segment); and blue is the transverse/sigmoid sinus. Copyright Walter C. Jean. Published with permission. Click here to view.

Zone 3 and the supratentorial portions of the tumors were not part of this study. Since translabyrinthine (TL) and AP were considered additions to RL, the blinded volumes for these were measured starting with the blinded volume for $\mathrm{RL}$ and eliminating the tumor that was exposed by adding the drilling of the labyrinth (TL) or Kawase's region (AP) (Fig. 3). 

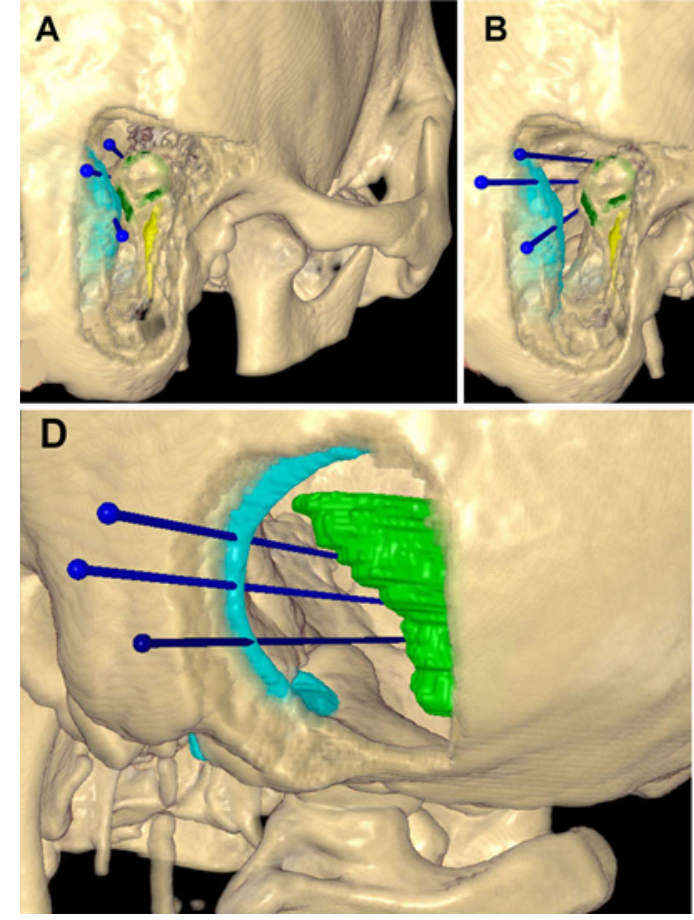

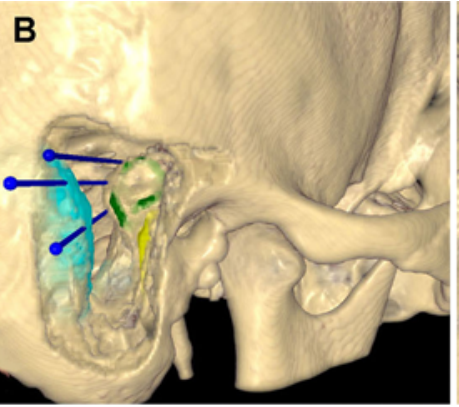

E

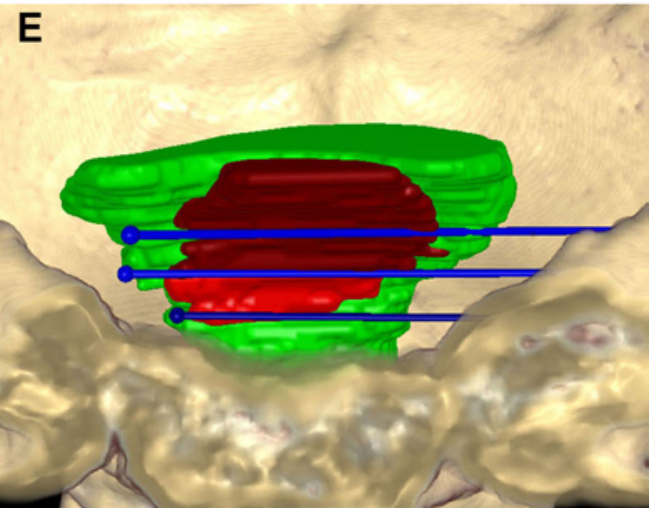

FIG. 2. A: Right-sided RL opening with dark blue lines of sight placed to determine regions of the tumor visually blocked by the bony labyrinth and other structures. B: Same specimen as in panel A, viewed from a different angle to show the lines embedded 1 $\mathrm{mm}$ into the sigmoid sinus. C: Posterior-to-anterior view of the petroclival region of the same specimen as in panels $A$ and $B$, demonstrating the segmentation of the blinded volume of the tumor which is not visualized by the RL approach. Orange is the zone 1 blinded volume; red is the zone 2 blinded volume; green is the semicircular canals; yellow is the facial nerve (mastoid segment); and light blue is the sigmoid sinus. D: Left-sided RS approach on a different specimen showing the dark blue lines of sight placed to determine regions of the tumor visually blocked by the brainstem. Again, the lines are embedded $1 \mathrm{~mm}$ deep into the sigmoid. E: Anterior-to-posterior view of the same specimen depicted in panel A, demonstrating the segmentation of the blinded volume of the tumor which is not visualized by the RS approach. Dark red is the zone 1 blinded volume; bright red is the zone 2 blinded volume; light green is the brainstem; and light blue is the sigmoid sinus.

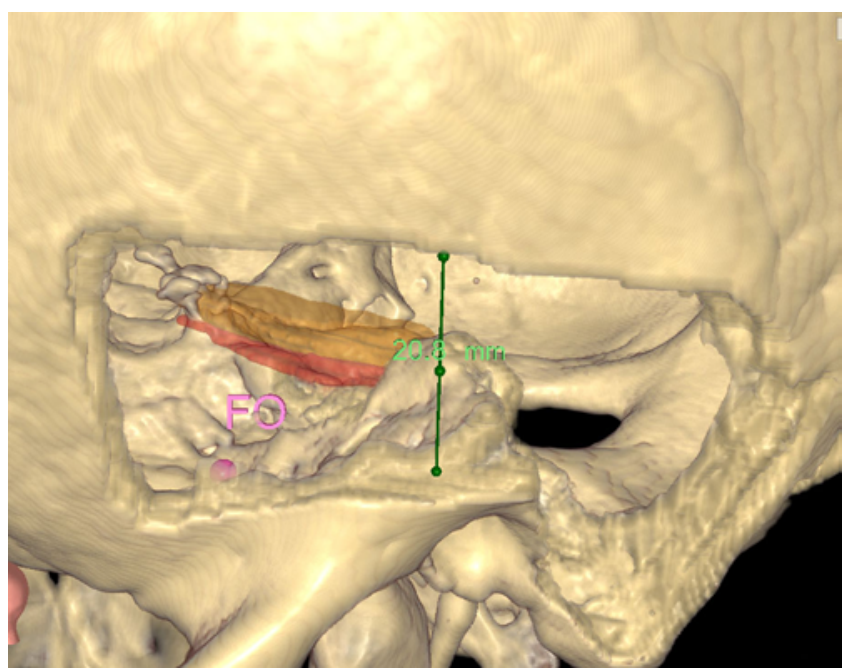

FIG. 3. View of the left subtemporal corridor showing the height of the temporal craniotomy. An AP has been drilled and reveals portions of the tumor (orange is zone 1; red is zone 2) that were part of the blinded volume of the RL approach (similar to Fig. $2 \mathrm{C}$, different specimen). The blinded volume of AP was calculated after erasure of the visualized tumor. $\mathrm{FO}=$ foramen ovale.

\section{Statistical Analysis}

Measurements made in the SRP system were compiled on a spreadsheet (Excel, Microsoft Corp.) for analysis. The mean and standard error of each set of measurements were calculated and the paired Student t-test was applied to evaluate for statistical significance between approaches; $\mathrm{p}$ values $<0.05$ were interpreted as indicating that a significant difference exists between data sets. Correlation of parameters was analyzed using the Spearman's correlation coefficient, with $\mathrm{p}<0.05$ considered statistically significant. The statistical methods employed were reviewed in consultation with a biostatistician.

\section{Results}

\section{The 15 Tumors}

Tumor size ranged from $9900 \mathrm{~mm}^{3}$ to $72,570 \mathrm{~mm}^{3}$ (SE 4587.3), and all traversed zones 1 and 2 of the petroclival region completely (i.e., from the dorsum sellae down to the level of the jugular foramen) (Fig. 4). Eight of the tumors had significant volumes in zone 3 , but because this region lies outside the definition of "true" petroclival meningioma, ${ }^{1}$ zone 3 was left out of the current study. In all cases, the basilar artery was displaced across 


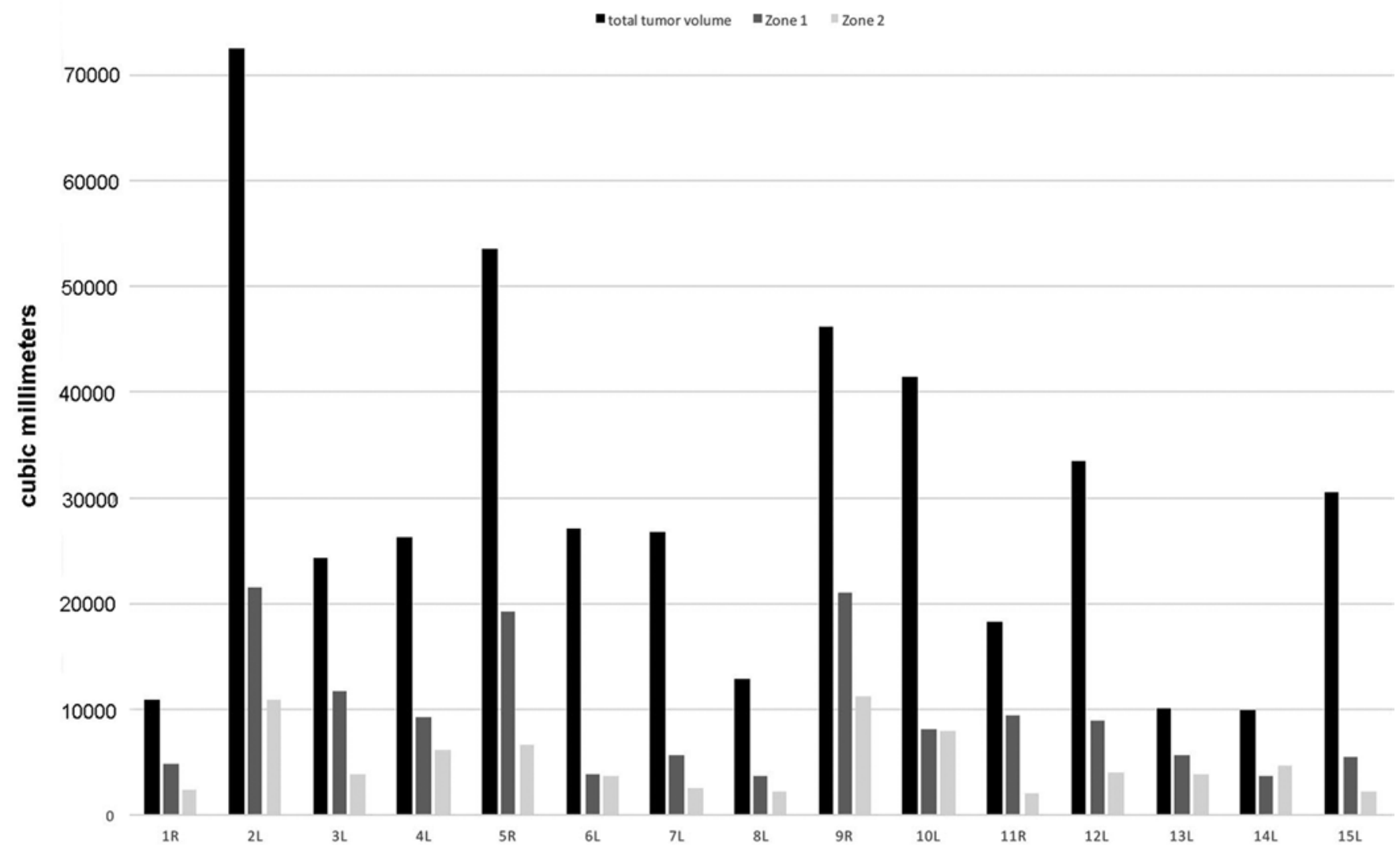

FIG. 4. Bar graph showing the total volume, as well as the volume by the petroclival zones, of the 15 tumors of the study. Zone 1 is the dorsum sellae to the center of the IAC, zone 2 is the center of the IAC to the center of the jugular foramen, and zone 3 is the center of the jugular foramen to the foramen magnum. ${ }^{6}$ Zones 1 and 2 are the focus of this study.

the midline and represented the most contralateral extent of the tumor. The PCA averaged $127.6^{\circ}$ (SE $3.43^{\circ}$, range $\left.97^{\circ}-142^{\circ}\right)$.

\section{SF Measurements}

Figure 5 shows the SFs at the 6 targets, but only when the approach exposed the target in at least $5(33.3 \%)$ of the 15 specimens. With RS, the targets of the ipsilateral PCF and midline clivus in both zones 1 and 2 were visible (Fig. $5 \mathrm{~A}$ ), with only one exception (specimen 9, zone $1 \mathrm{PCF}$ ). The brainstem blocked the view to the basilar artery in 3 specimens in zone 1 (80\% visible) and 5 in zone $2(67 \%$ visible).

The RL opening was unable to visualize the PCF in either zone. It was only able to visualize 2 of 15 targets $(13 \%)$ at the midline clivus in zone 1 . The target at the midline clivus in zone 2 corresponded to the CCD and this was visible via the RL in 5 specimens. The average SF here was only $74.9 \mathrm{~mm}^{2}$ (SE 21.5) (Fig. 5B). RL petrosectomy exposed the basilar artery in the majority of specimens $(93 \%$, zone $1 ; 80 \%$, zone 2$)$.

The TL approach failed to expose the PCF in either zone in nearly all specimens, but it was able to expose the midline clivus in most cases $(73 \%$, zone $1 ; 66 \%$, zone 2). The SFs are shown in Fig. 5C. The TL approach also exposed the basilar artery in the majority of cases $(93 \%$, zone $1 ; 87 \%$, zone 2 ).

AP provided the best exposure to all targets in zone 1 (Fig. 5D); in zone 2, it only exposed the PCF in 1 case and the midline clivus in 5 cases. For AP, the exposure in zone 2 improved moving from ipsi- to contralateral, and $93 \%$ of basilar artery targets were well exposed.

Comparing the SFs of the RS with the various transpetrosal approaches, the statistically significant $(\mathrm{p}<0.05$, paired Student t-test) relationships are shown in Table 1. In zone 1, the exposure of RS is significantly better than RL and TL for targets close to the side of the tumor, but it is worse than AP exposure at the PCF. In zone 2, RS again provided better exposure to the $\mathrm{PCF}$ and the midline clivus compared with all 3 petrosal approaches, but at the basilar artery, there was no significant difference.

The PCAs did not correlate with the SFs generated by the approaches through the petrous bone. They did, however, show a moderate correlation with the RS exposures to the basilar artery (zone $1, \mathrm{r}=0.56, \mathrm{p}=0.03$; zone $2, \mathrm{r}=$ $0.76, \mathrm{p}=0.02)$ and the PCF in zone $1(\mathrm{r}=0.63, \mathrm{p}<0.05)$. Confirming findings from previous studies,${ }^{14}$ the PCA predicted the visibility of the CCD through the RL approach. When the angle was $<140^{\circ}$, the CCD was visible in 1 of 10 specimens, but at $\geq 140^{\circ}$, it was exposed in 4 of 5 specimens $(\mathrm{p}=0.007$, chi-square test). 

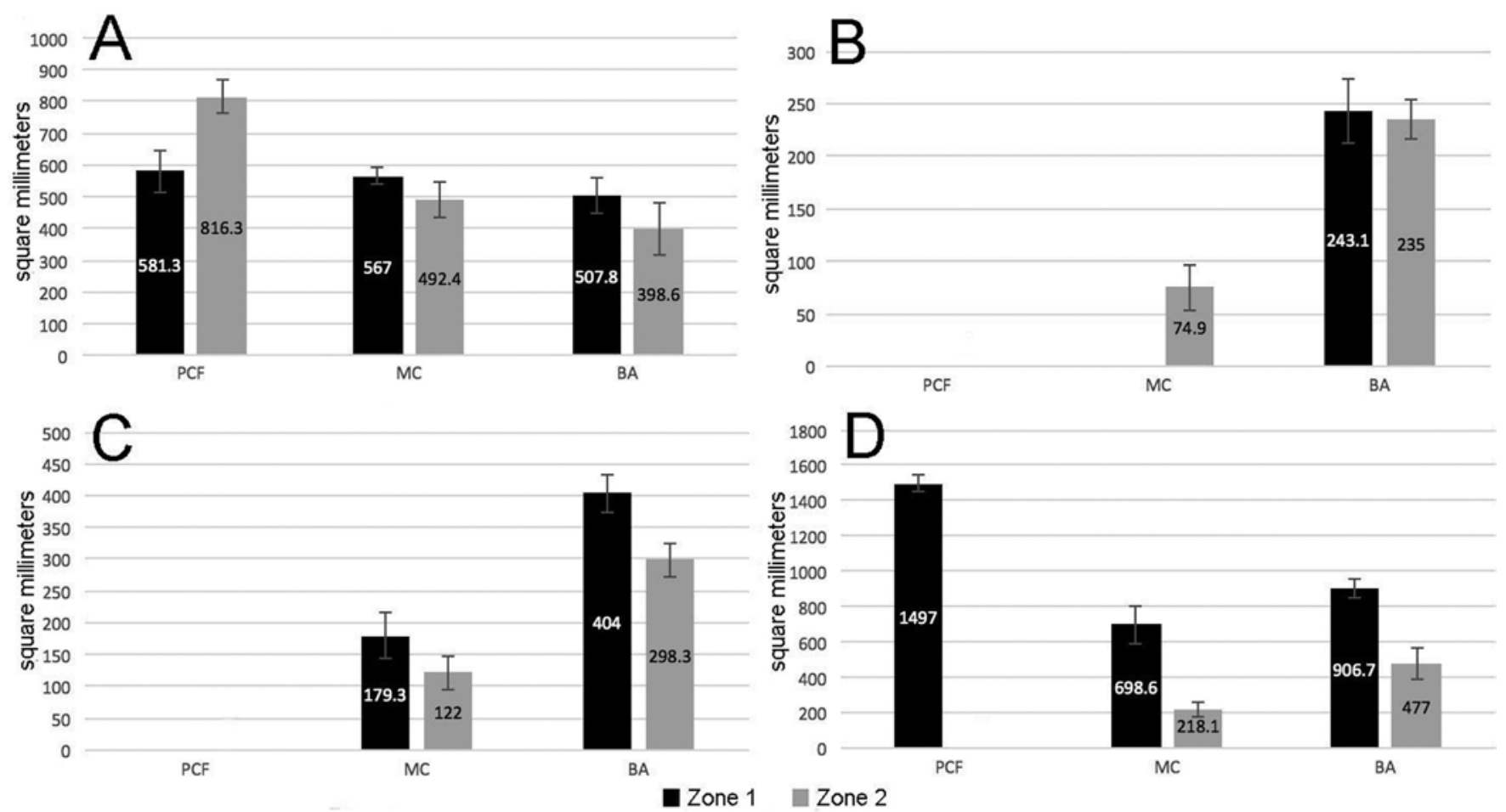

FIG. 5. The average SF at the 6 targets generated by the approaches. Measurements are shown only if the approach visualized the target in $>33 \%$ of all specimens. A: RS; B: RL; C: TL; and D: AP. For panel D, the SFs were measured through the subtemporal corridor. $\mathrm{MC}=$ midline clivus .

\section{Blinded Volumes}

Through the RS opening, the tumor component that was inaccessible (i.e., the blinded volume) averaged 489.3 $\mathrm{mm}^{3}$ in zone 1 and $173.3 \mathrm{~mm}^{3}$ in zone 2 (Fig. 6). In $87 \%$ of the specimens, all of this was hidden by the brainstem. Blinded volume obscured by bone on the petroclival side was only encountered in 2 cases and contributed less than $5 \%$ to the averages in both zones. The total average blinded volume had a moderate, inverse correlation with the total tumor size $(r=0.70, p<0.05)$, but the PCA showed no correlation with the blinded volumes for the RS.

Through the RL opening, the average blinded volume was $1316.7 \mathrm{~mm}^{3}$ in zone 1 and $1077.3 \mathrm{~mm}^{3}$ in zone 2 . Both were significantly larger than the corresponding volumes for RS ( $<<0.001$, paired Student t-test for both) (Table 2).
But unlike RS, the blinded portion was on the clival/dural side; only 1 specimen showed a minute portion on the pial or brainstem side, amounting to less than $3 \%$ of the total average RL blinded volume. By removing the TL, the blinded volume dropped significantly to an average of $363.3 \mathrm{~mm}^{3}$ in zone 1 and $483.3 \mathrm{~mm}^{3}$ in zone 2 (both compared with $\mathrm{RL}, \mathrm{p}<0.001$, paired Student t-test). The average reduction of the blinded volume was significantly higher in zone 1 $\left(945.3 \mathrm{~mm}^{3}\right)$ than in zone $2\left(589.3 \mathrm{~mm}^{3}\right)(\mathrm{p}=0.004$, paired Student t-test).

When AP was combined with the RL approach, using both corridors, the average blinded volume was $32.7 \mathrm{~mm}^{3}$ in zone 1 and $360 \mathrm{~mm}^{3}$ in zone 2 (both compared with RL, $\mathrm{p}<0.001$, paired Student t-test). The reduction of the total average blinded volume was significantly greater for AP ( $p$ $=0.005$ ), but this was almost entirely based on zone 1 , as

TABLE 1. Pairwise comparisons of SF at the 6 targets generated by each approach

\begin{tabular}{lcccc}
\hline & Retrosigmoid & Retrolabyrinthine & Translabyrinthine & Anterior Petrosectomy \\
\hline Retrosigmoid & & RS; PCF, MC & RS; PCF, MC & RS; PCF, MC \\
\hline Retrolabyrinthine & RS; all 3 targets & & TL; MC, BA & AP; BA* \\
\hline Translabyrinthine & RS; PCF, MC & TL; MC, BA & & AP; BA* \\
\hline Anterior petrosectomy & AP; PCF, BA & AP; all 3 targets & AP; all 3 targets & \\
\hline
\end{tabular}

$\mathrm{BA}=$ basilar artery; $\mathrm{MC}=$ midline clivus.

The bottom left of the table relates to zone 1 and the top right relates to zone 2 (values in boldface type). The approaches listed in the table have statistically significantly larger $S F(p<0.05$ on paired Student $t$-test).

* No statistically meaningful comparison feasible at other targets. 


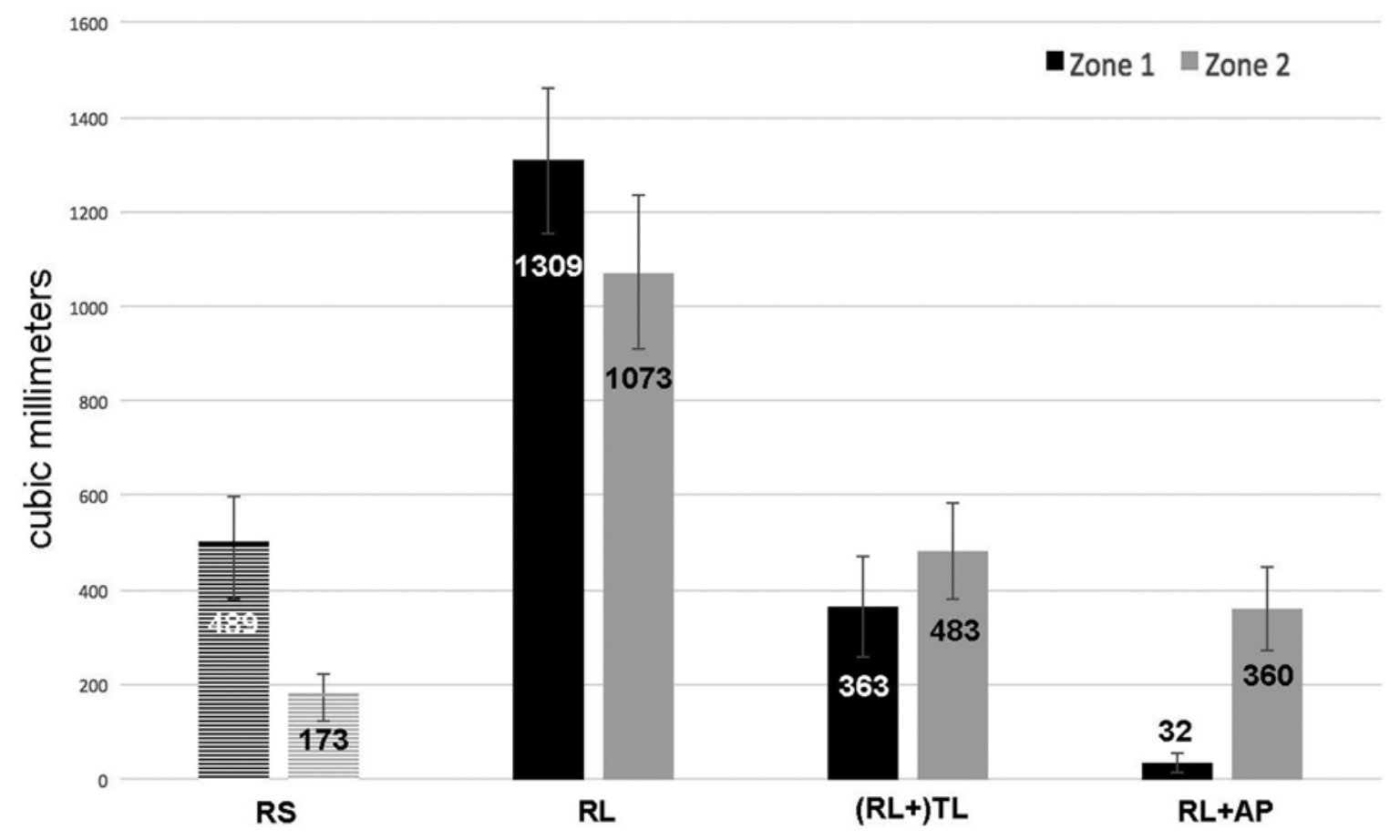

FIG. 6. Bar graph showing the average blinded portions of tumor as related to each approach. Stripe-filled columns denote blinded volume on the pial or brainstem side. Solid-filled columns denote blinded volumes on the dural or petroclival side.

that reduction was not significantly different for TL versus AP in zone 2.

Comparing RS and the add-ons for the RL approach, the total average blinded volume was not significantly different between RS and TL, or RS and AP. However, examining zones 1 and 2 separately, RS had less blinded volume in zone $2\left(173.3 \mathrm{~mm}^{3}\right)$ than TL $\left(483.3 \mathrm{~mm}^{3}\right)(\mathrm{p}=$ 0.02, paired Student t-test), and AP had less in zone 1 (32.7 $\mathrm{mm}^{3}$ ) than RS $\left(489.3 \mathrm{~mm}^{3}\right)$ (Table 2).

Finally, there was no correlation between total tumor size and blinded volumes of any approach through the petrous bone. The PCA showed only a weak correlation with the blinded volume in zone 2 for the RL approach $(\mathrm{r}=$ $0.36, \mathrm{p}<0.03)$.

\section{Discussion}

\section{Comparing VR and Cadaveric Studies}

VR turned the clinical data from patients into virtual models that we used in our laboratory experiments, and we compared tumor exposure in the petroclival region generated by various approaches. Our data cannot be applied retrospectively to the 15 patients whose anatomical data were used, since the choice of approach for each patient is multifaceted, ${ }^{16}$ and the exposure of the petroclival region is only one of them. Our data are therefore clinically applicable only for future patients for whom petroclival exposure is an important factor.

In their study of the petroclival region, Safavi-Abbasi et al. inflated tiny balloons in the surgical corridors to investigate how tumors might displace the brainstem and affect the exposure. ${ }^{17}$ Indeed, all previous studies on this topic have been done in cadavers (without tumors), and the distortion effect of tumors on normal structures can only be estimated..$^{3-5,18}$ The anatomical data in the current study eliminate any estimation, as the VR patient-based tumor models take into account any tumor alterations of the corridor.

TABLE 2. Pairwise comparisons of blinded volume inaccessible to each approach

\begin{tabular}{lcccc}
\hline & Retrosigmoid & Retrolabyrinthine & Translabyrinthine & Anterior Petrosectomy \\
\hline Retrosigmoid & & RS $p<0.001$ & RS $p=0.02$ & NS \\
\hline Retrolabyrinthine & RS $p<0.001$ & & TL $p<0.001$ & AP $p<0.001$ \\
\hline Translabyrinthine & NS & TL $p<0.001$ & & NS \\
\hline Anterior petrosectomy & AP $p=0.002$ & AP $p<0.001$ & AP $p=0.01$ & \\
\hline
\end{tabular}

NS = not significant.

The bottom left of the table relates to zone 1 and the top right relates to zone 2 (values in boldface type). The approaches listed in the table have statistically significantly smaller blinded volumes ( $p$ values: paired Student $t$-test). 
However, VR models do have similar limitations as cadavers. Regardless of whether the brain is fresh or fixed, cadaveric studies can only estimate the brain's impact on exposure. Cadaveric brains cannot simulate the pliability and turgidity of the brain in a patient, since those factors are influenced during surgery by the release of cerebrospinal fluid and hyperventilation, among many other maneuvers. Moreover, the brains in cadavers can be retracted with massive forces without physiological consequences.

Similarly, VR cannot account for the impact of the brain on exposure. But by limiting the size of the bone opening, we hoped to minimize the brain's impact on exposure. In this study, the RS craniotomy was made no wider than 20 $\mathrm{mm}$ and the middle fossa craniotomy no taller than the same. We assumed that the cerebellum and temporal lobe cannot be retracted beyond $20 \mathrm{~mm}$, and we placed our lines of sight against the bony margin at the edge of these small openings.

\section{The Extended RS Approach}

The designation "extended" pertains to placement of the opening at the edge of the sigmoid sinus. With a width of only $20 \mathrm{~mm}$, the aperture of our extended RS is quite small, and we chose this approach because of advantages that were well documented in previous studies. ${ }^{10-12}$ From our data, this afforded large SFs to most targets, and the inaccessible portions were very small, amounting to only $0 \%$ to $10 \%$ of the total tumor volume. One particularly interesting finding was that total tumor size was inversely correlated to the blinded volume, confirming what many experts have claimed in the past, that the tumor expands the corridor for the surgeon. However, the majority of the blinded volume was on the brainstem side, so if dissection of the tumor/brainstem pial interface is the single most important consideration, then additional corridors need to be considered.

\section{The RL Approach}

With this approach, the CCD was visualized in only $10 \%$ of specimens when the PCA was $<140^{\circ}$. However, when the PCA was $\geq 140^{\circ}$, the CCD was visualized in $80 \%$ ( $p=0.007$, chi-square test). This confirms the finding reported previously by Cohen et al. ${ }^{14}$ Moreover, our data also confirmed the findings by Abdel Aziz et al., ${ }^{6}$ as the blinded volume in zone 2 (i.e., where the CCD resides) correlated with the PCA $(r=0.56, p=0.03)$. The bony labyrinth significantly obstructed the view of the tumor on the (clival) dural side, and the blinded volumes were significantly higher for RL compared with all other approaches. However, the RL corridor did offer the advantage of a clear view of the tumor/brainstem pial surface, and only in one of our specimens was there a small (specimen 8, 190 $\mathrm{mm}^{3}$ ) inaccessible tumor portion on the pial side.

\section{Labyrinthectomy and AP as Modifications of the RL Platform}

Both TL and AP were effective to reduce the total blinded volume of RL to volumes that were insignificantly different from RS (TL vs RS, $\mathrm{p}=0.5$; AP vs RS, $\mathrm{p}=0.2$ ). The combined petrosal approach (i.e., RL and AP) had the smallest total average blinded volume, even though there was no significance compared with RS. Added benefits of the combined approach are the ability to resect the tumor with two corridors and a more perpendicular line of sight of the cranial nerves (compared with RS) pushed laterally.

\section{Limitations}

AP can of course be used separate from RL and TL approaches, but we did not study the inaccessible volume for AP as a stand-alone approach. Since its exposure of zone 2 is extremely limited, AP is probably not an appropriate choice as a stand-alone approach for large petroclival meningiomas that completely traverse zones 1 and 2 .

The VR models used in this study were completely dependent on the imaging data, and structures invisible on CT or MRI, such as cranial nerves, cannot be accounted for. Maneuvers that might mobilize obstacles, such as the transverse/sigmoid sinus junction, ${ }^{13,19}$ that are fixed in this study cannot be simulated in VR. And as a laboratory study, our findings cannot predict the surgical morbidity or clinical outcome for the patients. Furthermore, the tumor portion superior and inferior to the petroclival region (i.e., the middle fossa and foramen magnum) were not part of this study but may have a large impact not only on the choice of approach but on clinical outcome as well.

Although we studied the effect of adding TL and AP on the platform of RL, we did not specifically study the combination of RS and RL as this combination is seldom used. However, the data in this study suggest that this uncommon combination (i.e., RS and RL) should in fact be more frequently considered. ${ }^{20,21}$ The tumors' displacement of the brainstem created large corridors for the RS approach. The inaccessible portion of the tumor for this approach is on the pial/brainstem side, and since the exposure of this part is the strength of all the transpetrosal variants, the combined RL petrosectomy and RS approach would have no blind spots at all.

With 15 specimens, the size of the current study is small. For future considerations, large sample sizes, automated segmentation of structures, and machine learning algorithms can potentially lead to computer-generated suggestions of the best approach in order to help surgeons make strategic choices.

\section{Conclusions}

This is the first study of its kind, using patient CT and MRI data to generate 3D VR models for a laboratory study comparing surgical approaches. For approaches through the petrous bone, significant residual tumors seem inevitable with RL, and with the common modifications of this approach, such as adding labyrinthectomy (TL) or combining it with AP, our data show that the inaccessible portions of the tumors were significantly reduced but not eliminated. Since it is impossible to perform various approaches in separate surgeries in patients for comparison, VR offers a viable alternative for such comparative investigations.

\section{Acknowledgments}

We thank Peter Jih Cheng Wong for his assistance in data transfer between Beijing and Washington, DC. 


\section{References}

1. Almefty R, Dunn IF, Pravdenkova S, Abolfotoh M, Al-Mefty $O$. True petroclival meningiomas: results of surgical management. J Neurosurg. 2014;120(1):40-51.

2. Grossi PM, Nonaka Y, Watanabe K, Fukushima T. The history of the combined supra- and infratentorial approach to the petroclival region. Neurosurg Focus. 2012;33(2):E8.

3. Horgan MA, Anderson GJ, Kellogg JX, Schwartz MS, Spektor S, McMenomey SO, et al. Classification and quantification of the petrosal approach to the petroclival region. $J$ Neurosurg. 2000;93(1):108-112.

4. Janjua MB, Caruso JP, Greenfield JP, Souweidane MM, Schwartz TH. The combined transpetrosal approach: anatomic study and literature review. J Clin Neurosci. 2017;41: 36-40.

5. Mandelli C, Porras L, López-Sánchez C, Sicuri GM, Lomonaco I, García-Martínez V. The partial labyrinthectomy petrous apicectomy approach to petroclival meningiomas. A quantitative anatomic comparison with other approaches to the same region. Neurocirugia (Astur). 2008;19(2):133-142.

6. Abdel Aziz KM, Sanan A, van Loveren HR, Tew JM Jr, Keller JT, Pensak ML. Petroclival meningiomas: predictive parameters for transpetrosal approaches. Neurosurgery. 2000;47(1):139-152.

7. Jean WC, Tai AX, Hogan E, Herur-Raman A, Felbaum DR, Leonardo J, Syed HR. An anatomical study of the foramen of Monro: implications in management of pineal tumors presenting with hydrocephalus. Acta Neurochir (Wien). 2019; 161(5):975-983.

8. Tai AX, Herur-Raman A, Jean WC. The benefits of progressive occipital condylectomy in enhancing the far lateral approach to the foramen magnum. World Neurosurg. 2020;134: e144-e152.

9. Tai AX, Sack KD, Herur-Raman A, Jean WC. The benefits of limited orbitotomy on the supraorbital approach: an anatomical and morphometric study in virtual reality. Oper Neurosurg (Hagerstown). 2020;18(5):542-550.

10. Liebelt BD, Huang M, Britz GW. A comparison of cerebellar retraction pressures in posterior fossa surgery: extended retrosigmoid versus traditional retrosigmoid approach. World Neurosurg. 2018;113:e88-e92.

11. Quiñones-Hinojosa A, Chang EF, Lawton MT. The extended retrosigmoid approach: an alternative to radical cranial base approaches for posterior fossa lesions. Neurosurgery. 2006; 58(4)(suppl 2):ONS-208-ONS-214.

12. Raza SM, Quinones-Hinojosa A. The extended retrosigmoid approach for neoplastic lesions in the posterior fossa: technique modification. Neurosurg Rev. 2011;34(1):123-129.

13. Labidi M, Watanabe K, Hanakita S, Froelich SC. Tumors around the clivus: petroclival. In: Jean WC, ed. Skull Base Surgery: Strategies. 1st ed. Thieme; 2019:165-172.

14. Cohen PL, Abdel Aziz KM, Froelich SC, Keller JT, van Loveren HR. Predictors of operative exposure with frameless stereotactic guidance in the posterior petrosectomy. Paper presented at: 12th Annual Meeting of the North American Skull Base Society; March 4, 2001; Orlando, FL.

15. De Rosa A, Pineda J, Cavallo LM, Di Somma A, Romano A, Topczewski TE, et al. Endoscopic endo- and extra-orbital corridors for spheno-orbital region: anatomic study with illustrative case. Acta Neurochir (Wien). 2019;161(8):16331646.
16. Isolan GR, Wayhs SY, Lepski GA, Dini LI, Lavinsky J. Petroclival meningiomas: factors determining the choice of approach. J Neurol Surg B Skull Base. 2018;79(4):367-378.

17. Safavi-Abbasi S, Zabramski JM, Deshmukh P, Reis CV, Bambakidis NC, Theodore N, et al. Moving toward the petroclival region: a model for quantitative and anatomical analysis of tumor shift. J Neurosurg. 2007;107(4):797-804.

18. Sharma M, Ambekar S, Guthikonda B, Nanda A. A comparison between Kawase and extended retrosigmoid approaches (retrosigmoid transtentorial and retrosigmoid intradural suprameatal approaches) for accessing the petroclival tumors. A cadaveric study. J Neurol Surg B Skull Base. 2014;75(3): 171-176.

19. Kusumi M, Fukushima T, Mehta AI, Aliabadi H, Nonaka Y, Friedman AH, Fujii K. Tentorial detachment technique in the combined petrosal approach for petroclival meningiomas. $J$ Neurosurg. 2012;116(3):566-573.

20. Rassi MS, Zamponi JO Jr, Cândido DNC, Oliveira JG, Passos GAR, Borba LAB. Combined presigmoid and retrosigmoid approach to petroclival meningiomas. J Neurol Surg B Skull Base. 2018;79(5)(suppl 5):S402-S403.

21. Shitara S, Inoue T, Tanaka T, Hirai H, Fukushima T. Preoperative evaluation of the effects of sigmoid sinus ligation with both endovascular and open-field occlusion tests before removal of petroclival tumors. J Neurol Surg B Skull Base. 2020;81(2):193-197.

\section{Disclosures}

Dr. Jean: consultant for Surgical Theater. Mr. Srivastava: employed by Surgical Theater LLC as onsite senior clinical engineer for its VR system at George Washington University.

\section{Author Contributions}

Conception and design: Jean, Yang, Wu. Acquisition of data: Jean, Yang, Srivastava, Tai, Herur-Raman, Kim, Li. Analysis and interpretation of data: Jean, Yang, Tai, Kim. Drafting the article: Jean, Yang. Critically revising the article: Jean, Kim, Li, Wu. Reviewed submitted version of manuscript: Jean, Wu. Approved the final version of the manuscript on behalf of all authors: Jean. Statistical analysis: Jean. Administrative/technical/material support: Jean. Study supervision: Jean.

\section{Supplemental Information Videos}

Video 1. https://vimeo.com/558655765.

\section{Correspondence}

Walter C. Jean: George Washington University, Washington, DC. opus@alumni.princeton.edu. 